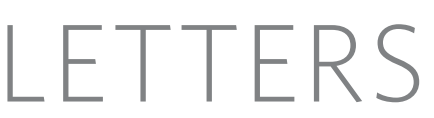

\title{
Epithelial NEMO links innate immunity to chronic intestinal inflammation
}

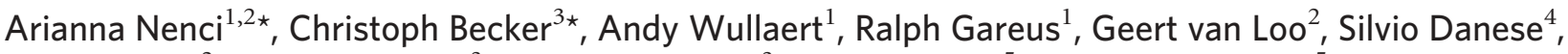 \\ Marion Huth ${ }^{2}$, Alexei Nikolaev ${ }^{3}$, Clemens Neufert ${ }^{3}$, Blair Madison ${ }^{5}$, Deborah Gumucio ${ }^{5}$, Markus F. Neurath ${ }^{3 \star}$ \\ \& Manolis Pasparakis ${ }^{1,2}$
}

Deregulation of intestinal immune responses seems to have a principal function in the pathogenesis of inflammatory bowel disease $^{1-4}$. The gut epithelium is critically involved in the maintenance of intestinal immune homeostasis-acting as a physical barrier separating luminal bacteria and immune cells, and also expressing antimicrobial peptides ${ }^{3,5,6}$. However, the molecular mechanisms that control this function of gut epithelial cells are poorly understood. Here we show that the transcription factor NFкB, a master regulator of pro-inflammatory responses ${ }^{7,8}$, functions in gut epithelial cells to control epithelial integrity and the interaction between the mucosal immune system and gut microflora. Intestinal epithelial-cell-specific inhibition of NF-кB through conditional ablation of NEMO (also called IкB kinase- $\gamma($ IKK $\gamma)$ )

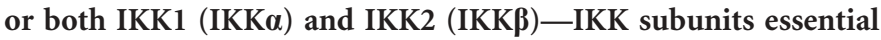
for NF- $\kappa$ B activation ${ }^{7-9}$-spontaneously caused severe chronic intestinal inflammation in mice. NF- $\kappa B$ deficiency led to apoptosis of colonic epithelial cells, impaired expression of antimicrobial peptides and translocation of bacteria into the mucosa. Concurrently, this epithelial defect triggered a chronic inflammatory response in the colon, initially dominated by innate immune cells but later also involving $\mathrm{T}$ lymphocytes. Deficiency of the gene encoding the adaptor protein MyD88 prevented the development of intestinal inflammation, demonstrating that Toll-like receptor activation by intestinal bacteria is essential for disease pathogenesis in this mouse model. Furthermore, NEMO deficiency sensitized epithelial cells to tumour-necrosis factor (TNF)-induced apoptosis, whereas TNF receptor-1 inactivation inhibited intestinal inflammation, demonstrating that TNF receptor- 1 signalling is crucial for disease induction. These findings demonstrate that a primary NF- $\kappa B$ signalling defect in intestinal epithelial cells disrupts immune homeostasis in the gastrointestinal tract, causing an inflammatory-bowel-disease-like phenotype. Our results identify $\mathrm{NF}-\kappa \mathrm{B}$ signalling in the gut epithelium as a critical regulator of epithelial integrity and intestinal immune homeostasis, and have important implications for understanding the mechanisms controlling the pathogenesis of human inflammatory bowel disease.

To investigate the function of NF- $\kappa \mathrm{B}$ signalling in the gut epithelium in vivo, we generated mice lacking NEMO specifically in intestinal epithelial cells (NEMO ${ }^{\text {IEC-KO }}$ mice) by crossing mice carrying loxP-flanked (floxed, FL) NEMO (Ikbkg) alleles ${ }^{10}$ with villin-Cre transgenics ${ }^{11}$. Southern blot DNA analysis showed NEMO deletion specifically in the small intestine and colon of $\mathrm{NEMO}^{\mathrm{IEC}-\mathrm{KO}}$ mice (Fig. 1b). Furthermore, immunostaining showed lack of NEMO expression in most epithelial cells from the small intestine and colon
(Fig. 1a), indicating efficient NEMO ablation in intestinal epithelial cells.

$\mathrm{NEMO}^{\mathrm{IEC}-\mathrm{KO}}$ mice were born at the expected mendelian ratio; however, at a young age they showed runting, diarrhoea and rectal bleeding, indicating intestinal pathology. High-resolution miniendoscopy ${ }^{12}$ revealed signs of severe colitis with thickening of the bowel wall, a granular and bleeding mucosa, loss of regular vessel architecture and diarrhoea in NEMO ${ }^{\mathrm{IEC}-\mathrm{KO}}$ mice (Fig. 1c). Furthermore, macroscopic examination showed severe pancolitis affecting all parts of the colon distal from the caecum but sparing the small intestine (Fig. 1d). The colitis phenotype was observed in all $\mathrm{NEMO}^{\mathrm{IEC}-\mathrm{KO}}$ mice examined older than 3 weeks, irrespective of whether they were maintained in a specific pathogen-free or a conventional animal facility. Macroscopic and histological examination of 6-week-old NEMO ${ }^{\text {IEC-KO }}$ mice did not reveal any pathological findings in the small intestine, whereas the colon was heavily inflamed (Supplementary Fig. 1 and data not shown). Colitis in NEMO ${ }^{\mathrm{IEC}-\mathrm{KO}}$ mice was characterized by thickening of the mucosa, enlarged crypts with loss of goblet cells, and a marked infiltration of mononuclear cells into the mucosa and submucosa, similar to the histopathology seen in patients with inflammatory bowel diseases (Fig. 1e and Supplementary Fig. 2). Analysis of pro-inflammatory gene expression showed upregulation of $I l 1 b, I l 6, T n f$ and $C c l 2$ (also called MCP-1) in the colon of NEMO ${ }^{\mathrm{IEC}-\mathrm{KO}}$ mice already at 2 weeks after birth (Fig. 2a), which was even more pronounced in 6-week-old animals, when Ccl5 (RANTES) was also induced. To investigate which cells were responsible for the inflammatory response in the colon of $\mathrm{NEMO}^{\mathrm{IEC}-\mathrm{KO}}$ mice, we examined the cellular infiltrate within the mucosa. In 2-week-old mice, inflammatory infiltrates were dominated by large numbers of dendritic cells and granulocytes, and to a much lesser extent by $\mathrm{CD} 4{ }^{+} \mathrm{T}$ cells (Fig. 2b). However, at 12 and 36 weeks, colonic tissue from $\mathrm{NEMO}^{\mathrm{IEC}-\mathrm{KO}}$ mice showed numerous lymphoid follicles and a massive infiltration with dendritic cells, $\mathrm{CD}^{+}{ }^{+} \mathrm{T}$ cells and granulocytes (Fig. 2b). Thus, intestinal epithelialcell-specific NEMO ablation causes the spontaneous development of severe chronic colitis associated with increased expression of inflammatory cytokines and chemokines, an early accumulation of innate immune cells, and the presence of T-cell infiltrates at later time points.

NEMO-deficient cells lack the ability to activate NF- $\kappa \mathrm{B}$ and are sensitive to apoptosis induced by cytokines such as $\mathrm{TNF}^{10,13,14}$. Indeed, TdT-mediated dUTP nick end labelling (TUNEL) staining of colon cross-sections from 2-week-old mice revealed extensive epithelial cell apoptosis in a few scattered areas in $\mathrm{NEMO}^{\mathrm{IEC}-\mathrm{KO}}$ mice but

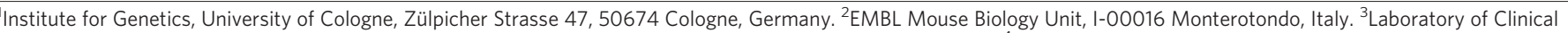

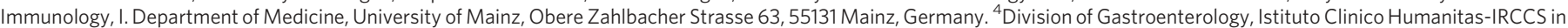

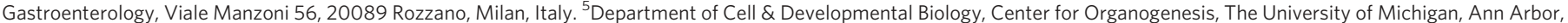
Michigan 48109-0616, USA

*These authors contributed equally to this work.
} 
not in controls (Fig. 3a). Furthermore, histological analysis of colon sections from these mice revealed the presence of crypts showing extensive epithelial destruction with loss of epithelial cells in such areas (Fig. 3b). Therefore, a localized disruption of epithelial integrity occurs in young $\mathrm{NEMO}^{\mathrm{IEC}-\mathrm{KO}}$ mice, which probably allows for the translocation of bacteria from the lumen into the mucosa. Consistently, fluorescence in situ hybridization (FISH) using a probe specific for the ribosomal RNA of all eubacteria showed the presence of bacteria within the lamina propria of NEMO ${ }^{\mathrm{IEC}-\mathrm{KO}}$ mice, in close proximity to areas displaying defects in epithelial integrity (Fig. 3c). High-power microscopic examination showed that in addition to the presence of free bacterial rods in the mucosa, cells in these areas also stained positive for bacterial rRNA, indicating phagocytosis of bacteria by immune cells (Fig. 3d). Immunofluorescence analysis revealed an accumulation of $\mathrm{CD}_{11 \mathrm{c}^{+}}$dendritic cells, neutrophils and a few $\mathrm{CD}^{+} \mathrm{T}$ cells around areas showing local disruption to a
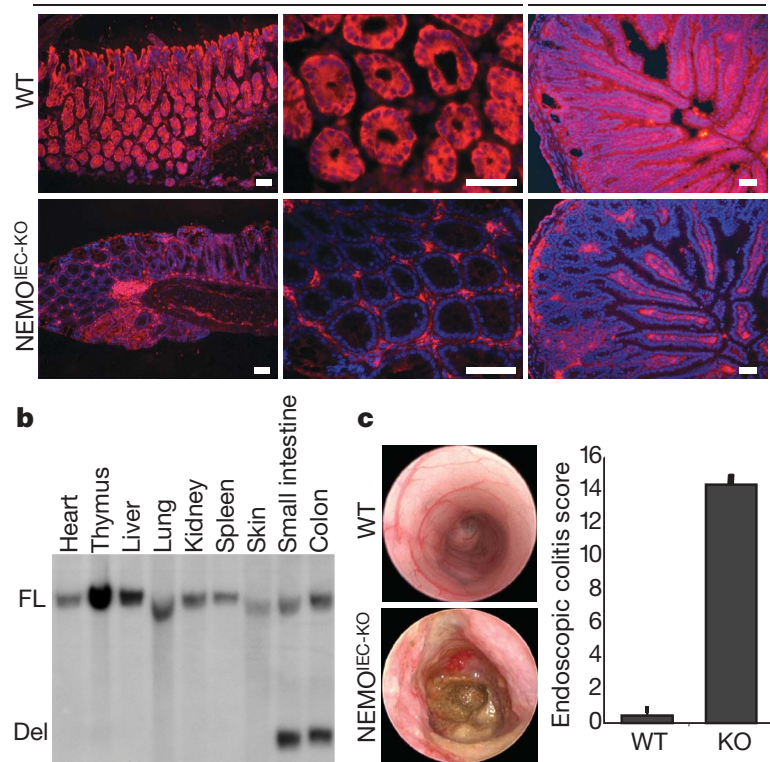

c

d

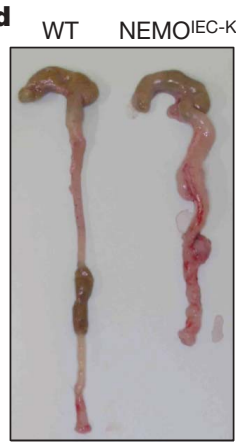

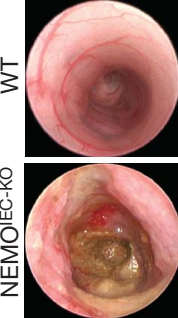

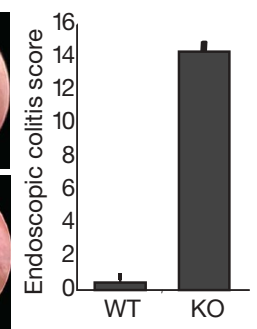

e

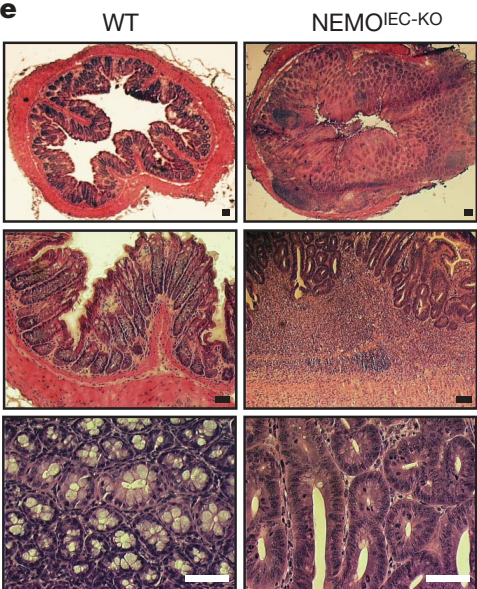

Figure 1 | Intestinal epithelium-specific NEMO ablation causes severe spontaneous colitis. a, Immunofluorescence with anti-NEMO antibodies shows efficient NEMO ablation in the intestinal epithelium of NEMO ${ }^{\text {IEC-KO }}$ mice. WT, wild type. $\mathbf{b}$, Southern blot DNA analysis shows NEMO deletion (Del) specifically in the intestines of $\mathrm{NEMO}^{\mathrm{IEC}-\mathrm{KO}}$ mice. c, $\mathrm{NEMO}^{\mathrm{IEC}-\mathrm{KO}}$ $(n=3)$ and wild-type $(n=5)$ mice were examined at the age of 32-36 weeks using mini-endoscopy. Murine endoscopic index of colitis severity (MEICS $)^{29}$ scores are shown (mean \pm s.d.). d, The colon of $\mathrm{NEMO}^{\mathrm{IEC}-\mathrm{KO}}$ mice is thickened and shortened, indicating severe colitis. e, Haematoxylin-andeosin-stained colon cross-sections show severe inflammation and loss of goblet cells in NEMO ${ }^{\mathrm{IEC}-\mathrm{KO}}$ mice. All scale bars, $50 \mu \mathrm{m}$. epithelial integrity, suggesting that bacterial translocation into the mucosa leads to immune cell recruitment and activation (Fig. 3e). Quantification of apoptotic cells ( $\mathrm{TUNEL}^{+}$), cells showing bacterial phagocytosis $\left(\mathrm{FISH}^{+}\right)$and of neutrophils $\left(\mathrm{MPO}^{+}\right)$in colon sections from newborn, 1-, 2- and 3-week-old mice showed that disruption of epithelial integrity, bacterial translocation into the mucosa and recruitment of neutrophils are first observed in 1-week-old mice and the pathology develops progressively over time (Supplementary Fig. 3 ). Taken together, our data are consistent with a model in which intestinal epithelial-cell-specific NEMO deficiency compromises colonic epithelial integrity, resulting in translocation of luminal bacteria into the mucosa. Concomitantly with bacterial translocation, an inflammatory response is initiated, characterized initially by activation of innate immune cells such as dendritic cells and neutrophils.
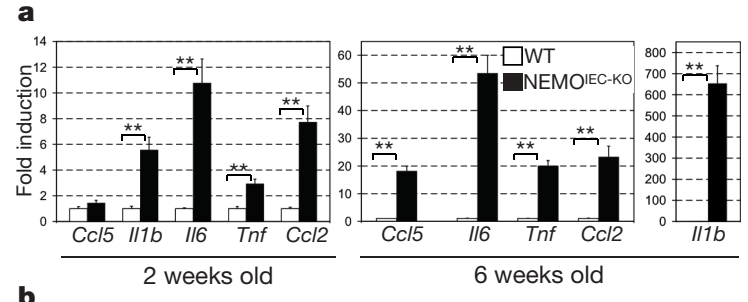

b
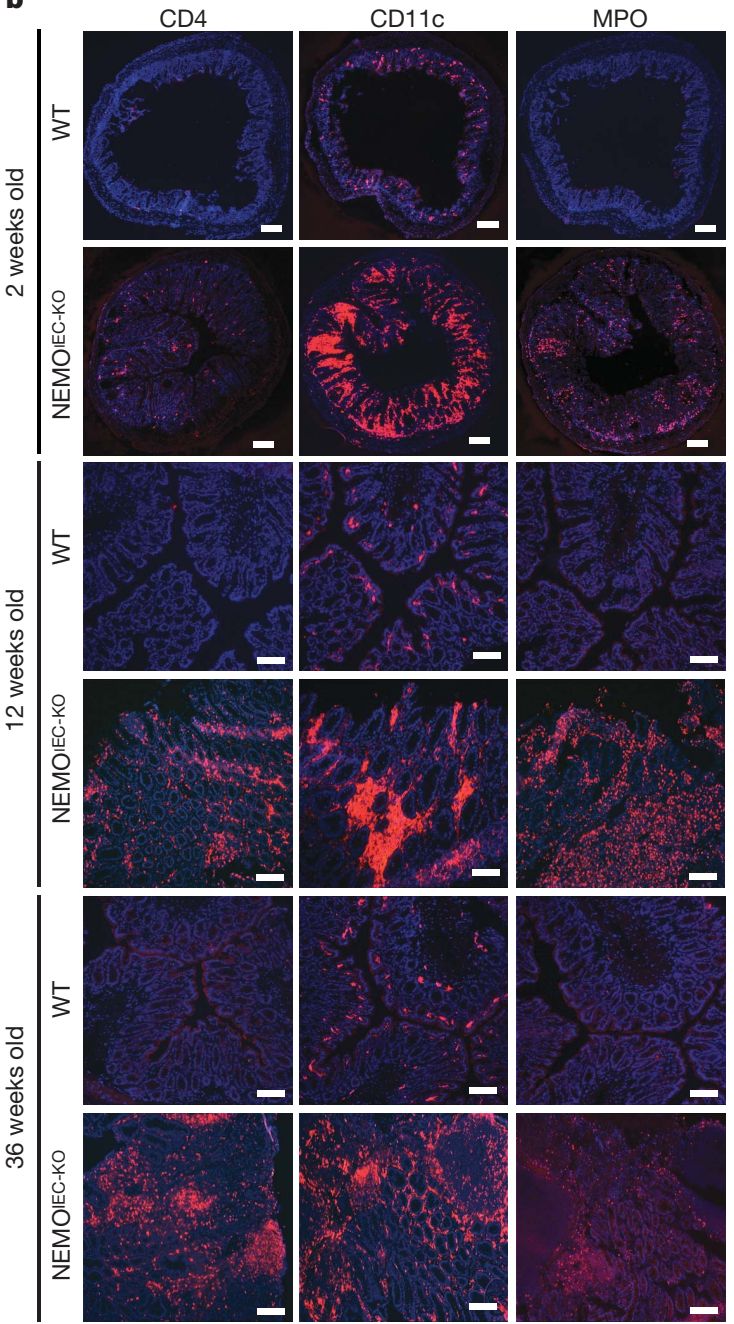

Figure 2 | Inflammation in the colon of NEMO'IEC-KO mice. a, Expression of pro-inflammatory cytokines and chemokines in the colons of $\mathrm{NEMO}^{\mathrm{IEC}-\mathrm{KO}}$ and wild-type mice ( $n=4-6$ mice per group). Data shown are mean values relative to ubiquitin ( \pm s.e.m.); double asterisk, $P<0.01$. b, Colon crosssections from NEMO ${ }^{\mathrm{IEC}-\mathrm{KO}}$ and wild-type mice were immunostained with antibodies for CD4, CD11c or myeloperoxidase (MPO) (red). Nuclei were counterstained with Hoechst 3342 (blue). Scale bars, $100 \mu \mathrm{m}$. 
This pathology starts in a localized fashion in scattered areas of the colon in young NEMO ${ }^{\text {IEC-KO }}$ mice between 1 and 2 weeks after birth. At later time points, the inflammation affects the entire colon and also involves $\mathrm{T}$ lymphocytes. In mice with advanced-stage colitis, we could detect lymphoid follicles surrounded by dendritic cells, whereas $\mathrm{CD} 11 \mathrm{c} / \mathrm{FISH}$ double staining revealed bacterial rRNA signals co-localized with dendritic cells in these areas (Fig. 3f),
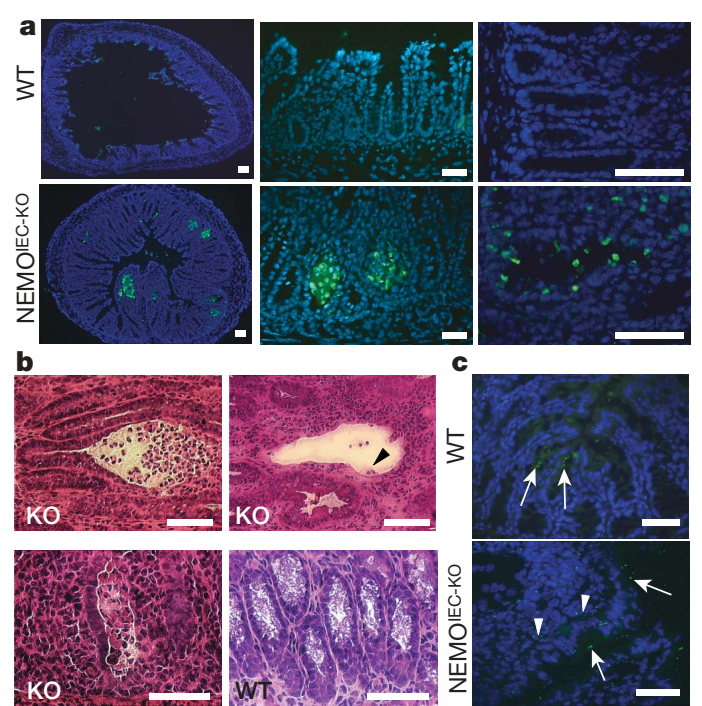

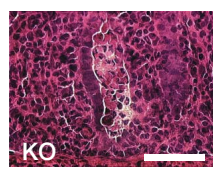

d

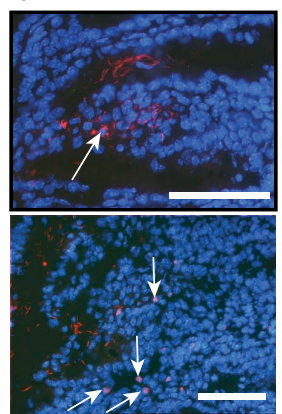

f
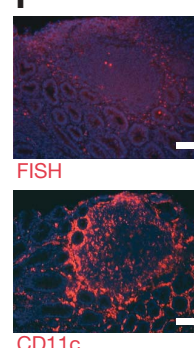

FISH sense control

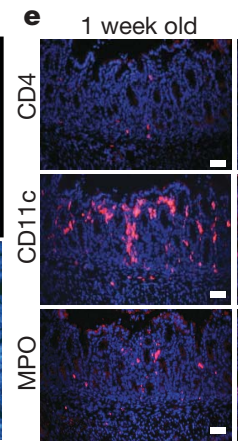

2 weeks old

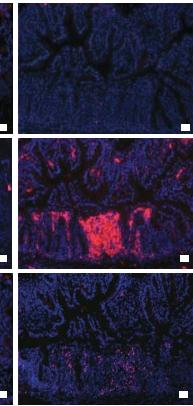

9
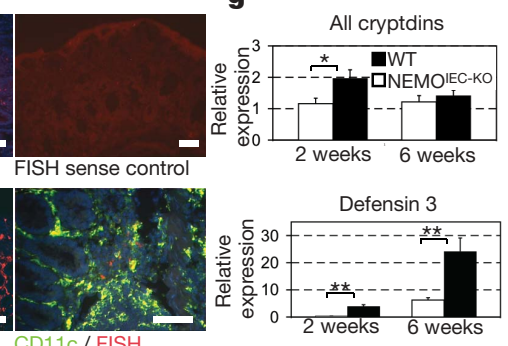

Figure 3 | Compromised epithelial integrity and bacterial translocation in the colon of NEMO ${ }^{\text {IEC-KO }}$ mice. a, TUNEL staining (green) on colon cross-sections from 2-week-old NEMO ${ }^{\text {IEC-KO }}$ and wild-type mice. b, Haematoxylin-and-eosin-stained colon cross-sections from 3-week-old $\mathrm{NEMO}^{\mathrm{IEC}-\mathrm{KO}}(\mathrm{KO})$ and wild-type mice. Note the epithelial disintegration in several crypts. The arrowhead depicts two remaining epithelial cells in a crypt showing nearly complete loss of epithelium. c, Bacterial FISH staining (green) was detected in the lamina propria of $\mathrm{NEMO}^{\mathrm{IEC}-\mathrm{KO}}$ colon (arrowheads), but was restricted to the lumen in wild-type mice (arrows). d, Bacterial FISH staining (red) co-localizes with cells in two representative $\mathrm{NEMO}^{\mathrm{IEC}-\mathrm{KO}}$ colon sections (see arrows). e, Focal infiltration of neutrophils and dendritic cells in the lamina propria of young NEMO ${ }^{\mathrm{IEC}-\mathrm{KO}}$ mice. f, Double FISH/CD11c staining on colon cross-sections from 36-week-old $\mathrm{NEMO}^{\mathrm{IEC}-\mathrm{KO}}$ mice shows co-localization of dendritic cells with bacteria around a lymphoid follicle. g, Expression of cryptdins and of defensin-3 in the colons of NEMO ${ }^{\text {IEC-KO }}$ and wild-type mice (4-5 mice per group). Results are shown as mean values relative to ubiquitin ( \pm s.e.m.); asterisk, $P<0.05$; double asterisk, $P<0.01$. All scale bars, $50 \mu \mathrm{m}$. indicating that the presence of bacteria drives the immune response also at later stages.

Antimicrobial peptide expression by the intestinal epithelium is believed to have an important function in controlling the number of bacteria around epithelial cells, and has recently been suggested to have a principal role in the pathogenesis of inflammatory bowel disease $^{6}$. Because the NF- $\kappa$ B signalling pathway regulates antimicrobial peptide expression ${ }^{6}$, we investigated whether NEMO deficiency affected antimicrobial peptide expression from the colonic epithelium. Using a primer set that detects simultaneously all 17 mouse cryptdin genes ${ }^{15}$, we found that collective expression of cryptdins was only mildly reduced in 2-week-old NEMO ${ }^{\mathrm{IEC}-\mathrm{KO}}$ mice compared to controls, and was unaffected at 6 weeks of age (Fig. $3 g$ ). However, beta-defensin-3, the expression of which is induced in mouse primary colonic epithelial cells by bacteria ${ }^{16}$, was significantly downregulated in 2- and 6-week-old NEMO ${ }^{\text {IEC-KO }}$ mice compared with littermate controls (Fig. 3g and Supplementary Fig. 4). A recent study reported that reduced expression of human beta-defensin-2, which is homologous to mouse beta-defensin-3, predisposes to colonic Crohn's disease in humans ${ }^{17}$. Thus, reduced expression of defensin-3 may lead to impaired antibacterial defence in the colon, and might be implicated in the translocation of commensal bacteria into the gut mucosa and consecutive colitis development in $\mathrm{NEMO}^{\text {IEC-KO }}$ mice.

Consistent with a previous report ${ }^{18}$, mice with intestinal epithelialcell-specific IKK2 ablation (IKK2 ${ }^{\mathrm{IEC}-\mathrm{KO}}$ ), generated by crossing villinCre transgenic mice with mice carrying loxP-flanked IKK2 (ikbkb) alleles $^{19}$, did not develop spontaneous intestinal inflammation (Supplementary Fig. 5). The differential effect of NEMO versus IKK2 ablation in the gut epithelium is probably due to the fact that pro-inflammatory-signal-induced NF- $\mathrm{KB}$ activity is completely blocked in NEMO-deficient cells but only partially affected in IKK2-deficient cells ${ }^{10,20}$. Indeed, NEMO-deficient intestinal epithelial cells displayed a complete lack of NF- $\kappa B$ activation, whereas IKK2deficient intestinal epithelial cells displayed low levels of residual NF- $\kappa \mathrm{B}$ activity (Fig. 4a and Supplementary Fig. 6). Furthermore, NEMO-deficient intestinal epithelial cells failed to upregulate NF$\kappa B$-dependent genes, such as I $\kappa B \alpha$ (also called Nfkbia) and IP10 (Cxcl10), upon in vivo lipopolysaccharide stimulation, whereas IKK2-deficient intestinal epithelial cells showed intermediate levels of induction compared to wild-type cells (Fig. 4b). Thus, NEMO deficiency completely blocks canonical NF- $\kappa \mathrm{B}$ activity in intestinal epithelial cells, whereas IKK2-deficient cells show residual NF- $\kappa B$ activation, presumably through compensatory signalling by means of IKK1. To test whether IKK1 signalling protects the colon of IKK $2^{\text {IEC-KO }}$ mice from intestinal inflammation, we generated mice lacking both IKK1 and IKK2 in intestinal epithelial cells (IKK1/ $2^{\mathrm{IEC}-\mathrm{KO}}$ ) by crossing the IKK2 ${ }^{\mathrm{IEC}-\mathrm{KO}}$ mice with mice carrying IKK1 (Chuk) loxP-flanked alleles ${ }^{21}$. Whereas intestinal epithelial-cellspecific IKK1-deficient mice (IKK1 $1^{\text {IEC-KO}}$ ) did not develop colitis, double IKK1/2 ${ }^{\mathrm{IEC}-\mathrm{KO}}$ mice displayed diarrhoea and rectal bleeding, as well as macroscopic, endoscopic and histological evidence of colitis (Fig. $4 \mathrm{c}-\mathrm{e}$ and Supplementary Fig. 7). Thus, although neither IKK1 ${ }^{\text {IEC-KO }}$ nor IKK 2 IEC-KO mice show spontaneous intestinal inflammation, $\mathrm{IKK} 1 / 2^{\mathrm{IEC}-\mathrm{KO}}$ mice develop severe colitis, similarly to $\mathrm{NEMO}^{\mathrm{IEC}-\mathrm{KO}}$ mice. These results demonstrate that complete inhibition of canonical NF- $\kappa \mathrm{B}$ activity in intestinal epithelial cells - achieved by ablation of either NEMO or both IKK1 and IKK2 - results in severe inflammatory colitis, and reveal that canonical IKK/NF- $\kappa \mathrm{B}$ signalling in intestinal epithelial cells has an essential function in maintaining epithelial integrity and immune homeostasis in the colon. Moreover, our findings uncover a redundancy between the two I $\kappa \mathrm{B}$ kinases in mediating this function.

The presence of bacteria within the lamina propria may trigger the development of chronic inflammatory colitis in NEMO ${ }^{\mathrm{IEC}-\mathrm{KO}}$ mice by means of Toll-like receptor (TLR) stimulation ${ }^{5}$. To investigate whether TLR activation contributes to the development of colitis 
in NEMO ${ }^{\text {IEC-KO }}$ mice, we crossed them with mice lacking MyD88, an adaptor molecule required for signalling by most members of the interleukin (IL)-1R/TLR family ${ }^{22}$. Macroscopic and immunohistological analysis of colons from $\mathrm{NEMO}^{\mathrm{IEC}-\mathrm{KO}} / \mathrm{MyD} 88$-deficient mice revealed no sign of colitis (13 mice were analysed between 6-20 weeks of age) (Fig. 4f), demonstrating that MyD88-dependent signals are essential for disease pathogenesis in this model. These results suggest that TLR-mediated bacterial recognition has a critical role in inducing intestinal inflammation in NEMO ${ }^{\mathrm{IEC}-\mathrm{KO}}$ mice; however, IL-1mediated signals that also depend on MyD88 might also be involved in the pathogenesis of colitis.

TNF blockade is an effective therapy for inflammatory bowel disease patients ${ }^{23}$. To investigate whether TNF signalling contributes to the development of colitis in NEMO ${ }^{\mathrm{IEC}-\mathrm{KO}}$ mice, we crossed them onto a TNF receptor-1 (TNFRI)-deficient background. Double NEMO $^{\text {IEC-KO } / T N F R I-d e f i c i e n t ~ m i c e ~ d i d ~ n o t ~ s h o w ~ m a c r o s c o p i c ~ o r ~}$ histological signs of colitis ( 10 mice were analysed between 6-10 weeks of age), demonstrating that TNFRI signalling is crucial for disease

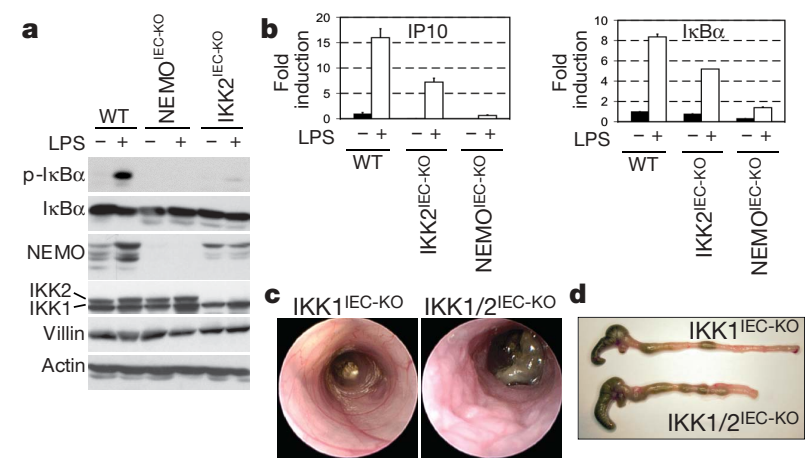

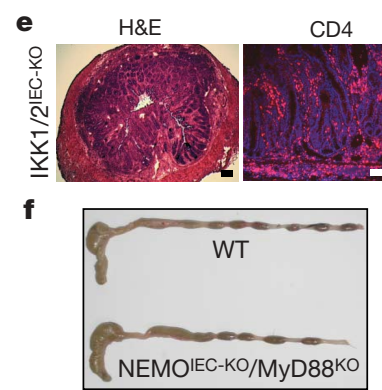

NEMOIEC-KO/MyD88KO

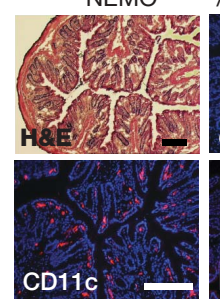

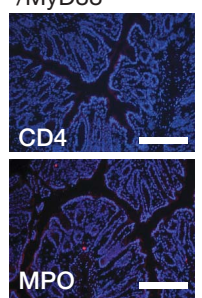

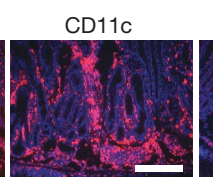

9

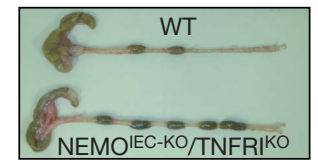

NEMOIEC-KO/TNFRIKO

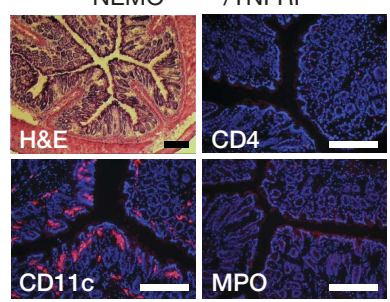

Figure 4 | NF- $к B$ inhibition in intestinal epithelial cells causes inflammatory colitis that depends on TNFRI and MyD88 signalling. $a$, b, Intestinal epithelial cells were isolated from mice with the indicated genotypes $90 \mathrm{~min}$ after administration of lipopolysaccharide (LPS; $25 \mu \mathrm{g}$ per $10 \mathrm{~g}$ body weight) or PBS. a, Immunoblot analysis of $\mathrm{I} \kappa \mathrm{B} \alpha$ phosphorylation and degradation, and NEMO, IKK2, villin and actin expression.

b, Quantitative RT-PCR analysis of $\mathrm{I} \kappa \mathrm{B} \alpha$ and IP10 expression. Mean values (土s.e.m.) are shown. c, d, Mini-endoscopy (c) and macroscopic colon examination (d) reveal severe colitis in 11 -week-old double IKK $1 / 2^{\text {IEC-KO }}$ mice but not in IKK1 ${ }^{\mathrm{IEC}-\mathrm{KO}}$ mice. e, Colon cross-sections from 11-week-old IKK $1 / 2^{\text {IEC-KO }}$ mice were stained with haematoxylin and eosin or with antibodies for CD4, CD11c or MPO (red). Nuclei were counterstained with Hoechst 3342 (blue). f, g, Macroscopic and histological examination of colons from 8-week-old NEMO ${ }^{\mathrm{IEC}-\mathrm{KO}} / \mathrm{MyD}^{\mathrm{KO}}(\mathbf{f})$ and $\mathrm{NEMO}^{\mathrm{IEC}-\mathrm{KO}}$ / TNFRI $^{\mathrm{KO}}(\mathbf{g})$ mice reveal lack of colon inflammation in both double mutant strains. All scale bars, $200 \mu \mathrm{m}$. pathogenesis in this model (Fig. 4g). Because cells lacking NEMO are more sensitive to TNF-induced apoptosis than cells lacking IKK2 (refs 10, 24), TNF-mediated killing of NEMO-deficient intestinal epithelial cells probably contributes to the induction of colon inflammation by compromising epithelial integrity in NEMO ${ }^{\mathrm{IEC}-\mathrm{KO}}$ mice. Indeed, TNF administration caused increased apoptosis of colon epithelial cells in $\mathrm{NEMO}^{\mathrm{IEC}-\mathrm{KO}} / \mathrm{TNF}$-deficient mice-generated by crossing with TNF-deficient mice ${ }^{25}$ and used here to facilitate assessment of apoptosis in a disease-free colon-compared with TNF-deficient, IKK2 ${ }^{\text {IEC-KO }}$ and wild-type mice (Supplementary Fig. 8), demonstrating that NEMO-deficient intestinal epithelial cells are more sensitive to TNF-induced killing in vivo than IKK2-deficient intestinal epithelial cells. These results suggest that one key mechanism by which TNF signalling induces colon inflammation in $\mathrm{NEMO}^{\mathrm{IEC}-\mathrm{KO}}$ mice is by killing the NF- $\mathrm{BB}$-deficient intestinal epithelial cells, resulting in disruption of epithelial integrity. However, TNF may also have a critical role in coordinating the mucosal inflammatory response by inducing the recruitment and activation of immune cells.

Taken together, our findings support a model (Fig. 5) in which impaired NF- $\kappa \mathrm{B}$ signalling in the colonic epithelium results in TNFmediated epithelial cell apoptosis and diminished expression of antimicrobial peptides, thus compromising epithelial integrity and allowing bacterial translocation into the mucosa of NEMO ${ }^{\mathrm{IEC}-\mathrm{KO}}$ mice. The presence of bacteria within the colonic mucosa activates innate immune cells through MyD88-dependent TLR signalling, triggering the expression of pro-inflammatory cytokines such as TNF and IL-1 $\beta$, which cause further destruction of the NF- $\kappa$ Bdeficient colonic epithelium and induce the recruitment of additional immune cells including T-lymphocytes, finally resulting in chronic inflammation and severe transmural pancolitis in $\mathrm{NEMO}^{\text {IEC-KO mice. }}$

Impairment of intestinal epithelial integrity and reduced cryptdin expression in humans have been suggested as important causative factors for the development of intestinal inflammation in patients with inflammatory bowel disease ${ }^{5,6}$. Our results with the $\mathrm{NEMO}^{\text {IEC-KO }}$ mice demonstrate that a primary epithelial defect resulting in impairment of both the integrity and antimicrobial defence of the gut epithelium can give rise to chronic intestinal inflammation. The rapid onset and high penetrance of the colitis phenotype in NEMO ${ }^{\mathrm{IEC}-\mathrm{KO}}$ mice suggest that a defect in epithelial $\mathrm{NF}-\kappa \mathrm{B}$ signalling may be an important proximal event in the pathogenesis of inflammatory bowel disease. Thus, epithelial NF- $\kappa \mathrm{B}$ deficiency may provide a new paradigm for the development of an inflammatory-bowel-disease-like disease. Furthermore, NF- $\kappa \mathrm{B}$ signalling in intestinal epithelial cells emerges as a crucial factor for

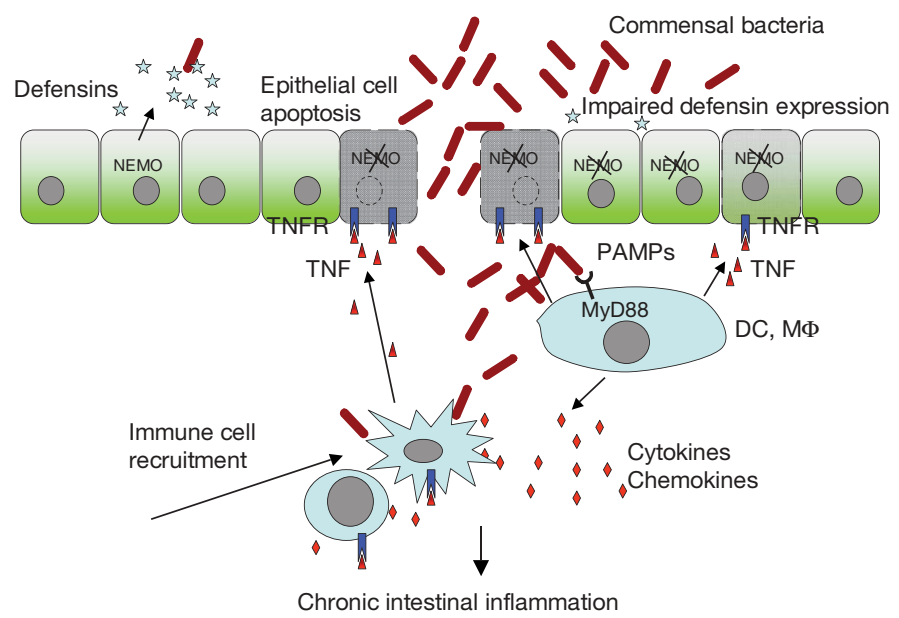

Figure 5 | NEMO-mediated NF- $\kappa$ B activation in intestinal epithelial cells is essential for the maintenance of epithelial barrier integrity and immune homeostasis in the colon. DC, dendritic cell; MФ, macrophage; PAMPS, pathogen-associated molecular patterns. 
maintaining homeostasis between commensal microflora and host immunity in the gastrointestinal tract, and represents a critical determinant for prevention of gut inflammation.

\section{METHODS}

Mice. The mouse lines used in this study have been described previously: $N E M O^{F L}$ (ref. 10), IKK2 ${ }^{F L}$ (ref. 19), villin-Cre ${ }^{11}$, TNFRI-deficient ${ }^{26}$, MyD88deficient $^{27}$, TNF-deficient ${ }^{25}, I K K 1^{F L}$ (ref. 21). The experiments were performed on mice backcrossed into the C57BL/6 genetic background for at least five generations. In all experiments, littermates carrying the loxP-flanked alleles but not expressing Cre recombinase were used as wild-type controls. The mice used in this study were housed in individually ventilated cage systems at the EMBL Mouse Biology Unit or at the Institute for Genetics of the University of Cologne, in either specific pathogen-free or conventional animal facilities.

Immunohistochemistry, FISH and apoptosis. Immunofluorescence of cryosections was performed using the TSA Cy3 system (Perkin Elmer) and a fluorescence microscope (Olympus fluorescence microscope) as described ${ }^{28}$. In situ hybridization of bacterial rRNA on glass slides was performed as previously described $^{28}$. Apoptosis was analysed using the in situ cell death detection kit (Roche).

Endoscopic procedures. For endoscopic colitis monitoring, a high-resolution mouse video endoscope, denoted Coloview, was used, as previously described ${ }^{29}$. Southern blot analysis. Southern blot analysis was performed as described previously ${ }^{10}$.

Isolation of intestinal epithelial cells. Intestinal epithelial cells were isolated from small intestines or colons (see Supplementary Information).

Immunoblot analysis. Total cell extracts were electrophoresed by SDSpolyacrylamide gel electrophoresis (10\%) and transferred to Immobilon P membranes (Millipore). Membranes were probed with the antibodies listed in the Supplementary Information.

Quantitative real-time PCR. Total RNA from colon, small intestine and intestinal epithelial cells was isolated using Trizol (Invitrogen) and analysed by realtime PCR with SyBr Green (Finnzymes) or by TaqMan gene expression (Applied Biosystems). Individual primer sequences are listed in the Supplementary Information. All values were normalized to the level of ubiquitin messenger RNA.

Received 8 December 2006; accepted 23 February 2007.

Published online 14 March 2007.

1. Bouma, G. \& Strober, W. The immunological and genetic basis of inflammatory bowel disease. Nature Rev. Immunol. 3, 521-533 (2003).

2. Hanauer, S. B. Inflammatory bowel disease: epidemiology, pathogenesis, and therapeutic opportunities. Inflamm. Bowel Dis. 12 (suppl. 1), S3-S9 (2006).

3. Macdonald, T. T. \& Monteleone, G. Immunity, inflammation, and allergy in the gut. Science 307, 1920-1925 (2005).

4. Neurath, M. F., Finotto, S. \& Glimcher, L. H. The role of Th1/Th2 polarization in mucosal immunity. Nature Med. 8, 567-573 (2002).

5. Abreu, M. T., Fukata, M. \& Arditi, M. TLR signaling in the gut in health and disease J. Immunol. 174, 4453-4460 (2005)

6. Wehkamp, J., Fellermann, K., Herrlinger, K. R., Bevins, C. L. \& Stange, E. F. Mechanisms of disease: defensins in gastrointestinal diseases. Nature Clin. Pract. Gastroenterol. Hepatol. 2, 406-415 (2005).

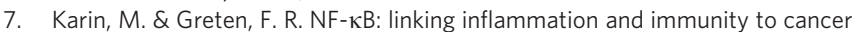
development and progression. Nature Rev. Immunol. 5, 749-759 (2005).

8. Li, Q. \& Verma, I. M. NF-KB regulation in the immune system. Nature Rev. Immunol. 2, 725-734 (2002).

9. Ghosh, S. \& Karin, M. Missing pieces in the NF-кB puzzle. Cell 109 (suppl.) S81-S96 (2002).

10. Schmidt-Supprian, M. et al. NEMO/IKK $\gamma$-deficient mice model incontinentia pigmenti. Mol. Cell 5, 981-992 (2000).
11. Madison, B. B. et al. Cis elements of the villin gene control expression in restricted domains of the vertical (crypt) and horizontal (duodenum, cecum) axes of the intestine. J. Biol. Chem. 277, 33275-33283 (2002).

12. Becker, C. et al. TGF- $\beta$ suppresses tumor progression in colon cancer by inhibition of IL-6 trans-signaling. Immunity 21, 491-501 (2004).

13. Rudolph, D. et al. Severe liver degeneration and lack of NF- $\mathrm{KB}$ activation in NEMO/IKK $\gamma$-deficient mice. Genes Dev. 14, 854-862 (2000).

14. Makris, C. et al. Female mice heterozygous for IKK $\gamma /$ NEMO deficiencies develop a dermatopathy similar to the human X-linked disorder incontinentia pigmenti. Mol. Cell 5, 969-979 (2000).

15. Darmoul, D. \& Ouellette, A. J. Positional specificity of defensin gene expression reveals Paneth cell heterogeneity in mouse small intestine. Am. J. Physiol. 271, G68-G74 (1996).

16. Lan, J. G. et al. Different cytokine response of primary colonic epithelial cells to commensal bacteria. World J. Gastroenterol. 11, 3375-3384 (2005).

17. Fellermann, K. et al. A chromosome 8 gene-cluster polymorphism with low human $\beta$-defensin 2 gene copy number predisposes to Crohn disease of the colon. Am. J. Hum. Genet. 79, 439-448 (2006).

18. Greten, F. R. et al. IKK $\beta$ links inflammation and tumorigenesis in a mouse model of colitis-associated cancer. Cell 118, 285-296 (2004)

19. Pasparakis, M. et al. TNF-mediated inflammatory skin disease in mice with epidermis-specific deletion of IKK2. Nature 417, 861-866 (2002).

20. Li, Q., Estepa, G., Memet, S., Israel, A. \& Verma, I. M. Complete lack of NF-кB activity in IKK1 and IKK2 double-deficient mice: additional defect in neurulation. Genes Dev. 14, 1729-1733 (2000).

21. Gareus, R. et al. Normal epidermal differentiation but impaired skin barrier formation upon keratinocyte-restricted IKK1 ablation. Nature Cell Biol. doi:10.1038/ncb1560 (in the press).

22. Kawai, T. \& Akira, S. TLR signaling. Cell Death Differ. 13, 816-825 (2006).

23. Targan, S. R. et al. A short-term study of chimeric monoclonal antibody cA2 to tumor necrosis factor $\alpha$ for Crohn's disease. Crohn's Disease cA2 Study Group. N. Engl. J. Med. 337, 1029-1035 (1997).

24. Luedde, $\mathrm{T}$. et al. Deletion of NEMO/IKK $\gamma$ in liver parenchymal cells causes steatohepatitis and hepatocellular carcinoma. Cancer Cell 11, 119-132 (2007).

25. Pasparakis, M., Alexopoulou, L., Episkopou, V. \& Kollias, G. Immune and inflammatory responses in TNF $\alpha$-deficient mice: a critical requirement for TNF $\alpha$ in the formation of primary B cell follicles, follicular dendritic cell networks and germinal centers, and in the maturation of the humoral immune response. J. Exp. Med. 184, 1397-1411 (1996).

26. Pfeffer, K. et al. Mice deficient for the $55 \mathrm{kd}$ tumor necrosis factor receptor are resistant to endotoxic shock, yet succumb to L. monocytogenes infection. Cell 73, 457-467 (1993).

27. Kawai, T., Adachi, O., Ogawa, T., Takeda, K. \& Akira, S. Unresponsiveness of MyD88-deficient mice to endotoxin. Immunity 11, 115-122 (1999).

28. Becker, C. et al. Constitutive $\mathrm{p} 40$ promoter activation and IL-23 production in the terminal ileum mediated by dendritic cells. J. Clin. Invest. 112, 693-706 (2003).

29. Becker, C. et al. In vivo imaging of colitis and colon cancer development in mice using high resolution chromoendoscopy. Gut 54, 950-954 (2005).

Supplementary Information is linked to the online version of the paper at www.nature.com/nature.

Acknowledgements We thank K. Pfeffer and S. Akira for providing TNFRI-deficient and MyD88-deficient mice, respectively. This work was supported by grants from the German Research Council to C.B. and M.F.N., and by EU-FP6 grants MUGEN and IMDEMI to M.P.; A.W. received a research fellowship from the Alexander von Humboldt Foundation.

Author Contributions A. Nenci, C.B., A.W., R.G, G.v.L, M.F.N and M.P. designed the research. A. Nenci, C.B, A.W, R.G, G.v.L, S.D., M.H., A. Nikolaev and C.N. performed the research. B.M. and D.G. contributed new reagents. A. Nenci, C.B., A.W., R.G, M.F.N and M.P. analysed the data and wrote the paper. M.F.N. and M.P share senior authorship.

Author Information Reprints and permissions information is available at www.nature.com/reprints. The authors declare no competing financial interests. Correspondence and requests for materials should be addressed to M.P. (pasparakis@uni-koeln.de). 